\title{
The differential use of monocular and binocular cues to depth in the perception of two trapezoid illusions
}

\author{
ROBERT ZENHAUSERN, FRANK DUFFY, and LESLEE NICKEL \\ St. John's University, Jamaica, New York 11499
}

\begin{abstract}
Twenty-four male and 24 female subjects were exposed to two dynamic trapezoid illusions under conditions of binocular and monocular regard. Distance and cues to perspective were also varied and the results on these two parameters were consistent with past research. In general, monocular regard resulted in significantly more illusory experiences than binocular regard. Binocular regard was not universally facilitating to veridical perception and, in fact, was detrimental for one-third of the subjects. There was strong evidence, moreover, that the effect of binocular cues was dependent on the particular illusion involved.
\end{abstract}

The rotating trapezoid illusion was first described by Ames (1951) as a demonstration of his transactional theory of perception. The perception of oscillation, through 100 to $120 \mathrm{deg}$, of a veridically rotating trapezoid was attributed to the "transaction" of the actual stimuli and the past experience of the observer. Ames reported that the force of the illusion increased with increasing cues to linear perspective, increased distance, and monocular viewing. Zegers and Murray (1962), in an extensive empirical investigation of the parameters of the illusion, verified most of Ames' findings. Increases in the cues to linear perspective and monocular viewing resulted in more illusory experiences. Distance and target size, however, were found to interact as the visual angle subtended by the target, with smaller visual angles resulting in the perception of more reversals.

Another interesting finding was the identification of three perceiver groups: (a) high, those who perceive a large number of reversals under almost all conditions; (b) intermediate (or distance), those who perceive an increasing number of reversals with increasing distance; and (c) low, those who perceive few reversals under almost all conditions. Cahill (1967) attempted to explain these three groups on the basis of relative dependence on the cues to linear perspective, but was unable to find a clear-cut relationship between this relative dependence and susceptibility to the illusion. Zenhausern (1969) attempted to explain her results on the basis of a differential dependence on other cues to depth in addition to linear perspective.

Zenhausern (1968) reported the discovery of a related illusion: the perception of rotation of an oscillating trapezoid. A comparison of the two illusions (Zenhausern, 1969) indicated a negative relationship

This paper is sponsored by Leonard Brosgole, who takes full editorial responsibility for its contents. between them, not only in terms of overall susceptibility, but also in terms of the parameters that affect their relative strengths. There was an overall correlation between the two illusions of -.63 , indicating that a subject who perceives a large number of illusory experiences on one illusion tends to perceive a small number on the other. The force of both illusions increased with increasing distance, but there was a differential effect of perspective cues on the two illusions. Increases in the cues to linear perspective increased the perception of oscillation of a veridically rotating target, but decreased the perception of rotation of a veridically oscillating target. In addition, there is preliminary evidence that speed of rotation of oscillation has a differential curvilinear effect on the two illusions.

Since the effect of binocular and monocular regard on the reverse illusion has not been studied, the purpose of this paper is to investigate that parameter. It is expected that more illusory experiences will be perceived under the monocular condition, but given the inverse relationship of other parameters of the two illusions, a differential effect would not be totally unexpected.

\section{METHOD}

\section{Subjects}

The subjects were 24 male and 24 female undergraduate students, ranging in age from 18 to 27 , with a median age of 20.9 years. All were volunteers and none had previous experience with either illusion.

\section{Apparatus}

The two targets were constructed of $1 / 8$-in.-thick aluminum coated with white acrylic daylight fluorescent paint, with no shading or other depth cues other than the target designs themselves. Although the two targets were identical with respect to outside dimensions, the internal configurations of windows and mullions were such that one target provided more cues to perspective than did the other (cf. Zenhausern, 1969). 


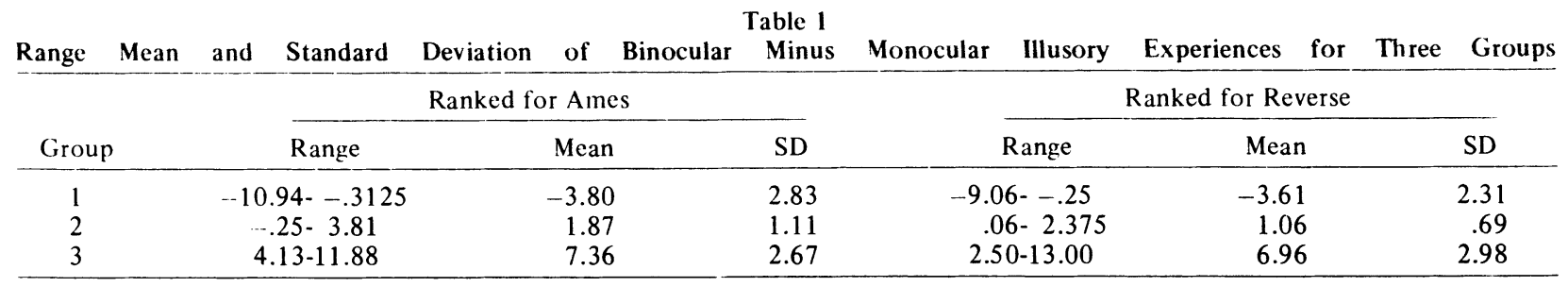

A constant-speed motor, rated at $31 \mathrm{rpm}$, was used to propel the targets. The drive transmission had two shafts, one for rotation through $360 \mathrm{deg}$ and one for oscillation through 120 deg. On the oscillation shaft the trapezoid oscillated 62 times/min. On the rotation shaft it rotated 31 times $/ \mathrm{min}$. An ITL-60 timer was used to control the length of the test period. The subjects were seated at a table to which was secured a chin rest. Responses were recorded on an electric counter which was activated by a hand-held microswitch. All the testing was conducted in a completely lightproof room which was painted matte black in order to preclude possible reflections. The targets were illuminated with an overhead ultraviolet light.

\section{Procedure}

There were 16 conditions in this experiment: rotation of the two targets at distances of 9 and $12 \mathrm{ft}$ viewed either binocularly or monocularly, and oscillation of the two targets with the small end in the frontal plane at distances of 9 and $12 \mathrm{ft}$ viewed either binocularly or monocularly. The presentation of the 16 conditions was counterbalanced among the 48 subjects in order to avoid a possible bias resulting from an order effect.

Each testing session consisted of 16 trials of 2-min duration each. There was a 1-min intertrial interval. Before each testing session each subject was dark adapted for $5 \mathrm{~min}$. Throughout the entire testing situation, dark adaptation was strictly maintained.

\section{RESULTS AND DISCUSSION}

All data reported were in terms of illusory experiences. In the case of the perception of oscillations with a veridically rotating target, it was the number of reported reversals. Since the targets were rotated at $31 \mathrm{rpm}$ for a total $2 \mathrm{~min}$ for each condition, the total number of possible reversals for any given trial was 124 . For the perception of rotation with a veridically oscillating target, the number of reported reversals was subtracted from 124, the actual number of veridical oscillations. Preliminary analysis of data was based upon a split-plot factorial analysis of variance with Motion, Perspective, Distance, and Visual Regard as within factors and Sex as a between factor.

The Distance main effect was significant $[F(1,47)=$ 4.38, $\mathrm{p}<.05]$, with the 9-ft distance $(\bar{X}=49.78)$ resulting in fewer illusory experiences than the 12 -ft distance $(\bar{X}=54.46)$. The lack of a Motion by Distance interaction indicates that the increased ambiguity, resulting from increased distance, was consistent for the two illusions. The significant Motion by Perspective interaction $[F(1,47)=6.04, p<.05]$ was consistent with past research in that the high perspective target resulted in more illusory experiences with the Ames illusion than the reverse illusion, while the opposite was true for the low perspective target.
Regard proved to be a significant factor $[F(1,47)=$ $14.56, \mathrm{p}<.01]$. Binocular viewing $(\bar{X}=48.70)$ resulted in significantly fewer illusory experiences than monocular viewing $(\overline{\mathrm{X}}=55.54)$. It is clear that binocular viewing provides more cues to veridical perception than monocular viewing, and the difference between the two is consistent with the notion that increases in ambiguity increase the force of the illusions. The lack of interaction between Regard and the two illusions seems to indicate a similarity in the relative importance of the perceptual cues for veridical perception.

While there were no sex differences in mean illusory experiences, male variability was greater on both illusions than female variability. An examination of the frequency distributions indicated that the males tended toward a bimodal distribution, with the majority of subjects reporting either a very small or a very large number of illusory experiences. The females, on the other hand, formed a distribution that approximated normality. Since the sample consisted of only 24 males and 24 females, this finding, while interesting, must be considered tentative.

In an effort to determine if the relative dependence on binocular cues was related to illusory perception, the number of illusory experiences reported for binocular regard was subtracted from the number of illusory experiences reported for monocular regard. As this number increased, the relative importance of the binocular cues could also be considered to increase. The results of this simple calculation were totally unexpected. The 48 subjects formed three groups, each of approximately 16 subjects. One group followed the expected pattern, with a fairly large positive difference between monocular and binocular regard. Another group showed very little difference. For the third group the difference was negative, that is, they reported more illusory experiences for binocular regard than monocular regard. It was as if the binocular cues were interfering with veridical perception.

The differences between monocular and binocular regard, obtained on both the Ames and reverse illusions and separated into the three groups, are shown in Table 1. Except for the fact that there were 17, as opposed to 16, subjects ranked on the Ames illusion, there was amazingly little overlap among the groupings. Either this differential use and misuse of binocular cues is a real phenomenon or the results of this experiment were unfortuitously misleading. (A replication is currently in progress to determine the reliability of the 
effect.) It is interesting to note that the middle groups, in which the binocular cues neither improve nor detract from veridical perception, have smaller ranges and standard deviations than the groups in which binocular cues help or hinder veridical perception. The variances of these two middle groups were significantly lower than those of the other groups.

Since subjects could be ranked separately on the Ames and reverse illusion in terms of the relative contribution of binocular and monocular cues, it was possible to correlate the two rankings. The resulting significant correlation of -.30 indicated that there was a tendency for subjects who use binocular cues to enhance veridical perception on one illusion to use them nonveridically on the other. This trend can be seen from Table 2 . Group 1 refers to those subjects who perceived more illusory experiences binocularly than monocularly; Group 2 refers to those subjects who showed little difference with respect to regard; and Group 3 refers to those subjects who showed the expected superiority of binocular regard. Group 1, ranked on either illusion, showed the largest positive difference between monocular minus binocular viewing, and this difference decreased through Groups 2 and 3. There was virtually no difference betwen monocular and binocular regard on one illusion for subjects who showed the greatest difference on the other illusion.

The differential use of perceptual cues has long been postulated to explain the difference with respect to susceptibility to the two illusions (cf. Cahill, 1967; Zegers \& Murray, 1962; Zenhausern, 1969). It has been assumed, however, that this differential dependence represented a fairly consistent perceptual style. The
Table 2

Mean Illusory Binocular and Monocular Experiences for One Illusion as a Function of Ranking on the Other

\begin{tabular}{|c|c|c|}
\hline \multirow{2}{*}{$\begin{array}{l}\text { Ranked on Ames } \\
\text { Group }\end{array}$} & \multicolumn{2}{|c|}{ Illusory Experiences on Reverse } \\
\hline & Monocular & Binocular \\
\hline 1 & 64.72 & 51.59 \\
\hline 2 & 59.80 & 55.09 \\
\hline 3 & 55.30 & 55.08 \\
\hline \multirow{2}{*}{$\begin{array}{l}\text { Ranked on Reverse } \\
\text { Group }\end{array}$} & \multicolumn{2}{|c|}{ Illusory Experiences on Ames } \\
\hline & Monocular & Binocular \\
\hline 1 & 50.91 & 36.55 \\
\hline 2 & 55.19 & 46.11 \\
\hline 3 & 46.84 & 47.78 \\
\hline
\end{tabular}

results of this experiment, however, point to a shift in the relative importance of cues as a function of the perceptual phenomenon involved.

\section{REFERENCES}

Ames, A. A. Visual perception and the rotating trapezoid window. Psychological Monographs, 1951, 65, No. 7(Whole No. 324).

CaHILl, M. Accuracy of position judgments of stationary targets yielded by three types of perceivers of the Ames trapezoid illusion. Unpublished doctoral dissertation, Fordham University, 1967.

Zegers, R. T., \& Murray, P. Perception of distortion: An experimental and clinical study. Technical Reports, 1962, NAVTRADEVCEN 506-1.

ZENHAUSERN, R. J. The perception of rotation with an oscillating trapezoid. Psychonomic Science, 1968, 13, 79-80.

ZENHAUSERN, R. J. Effect of perspective on two trapezoid illusions. Perceptual and Motor Skills, 1969, 28, 1003-1009.

(Received for publication April 7, 1976.) 